\title{
Incidence of complications of home parenteral nutrition in patients with cancer versus other disease groups
}

\author{
J. Gomez, K. Page and M. McAlindon \\ Nutrition Support Team, G Floor, Royal Hallamshire Hospital, Sheffield S10 2JF, UK
}

The range of diseases and situations where patients can benefit from parenteral nutrition (PN) is very wide, comprising benign to malignant conditions, and from short to long-term requirement of PN. This is an invasive technique, however, in which potential complications can be classified as nutritional or metabolic, catheter related, organ dysfunction, and psychological or social.

Technical and management improvements have allowed that long-term requirements of PN can be continued in the out-patient setting. Cancer is the commonest indication for home parenteral nutrition (HPN) in the USA and mainland Europe ${ }^{(1)}$, but it accounts for the much lower $11.7 \%$ in the UK ${ }^{(2)}$. The HPN service at the Sheffield Teaching Hospitals is therefore unusual within the UK in that approximately one third of our HPN patients have had cancer.

Advanced malignancy is likely to cause impaired immunity and disordered blood clotting, potentially leading to an increased risk of catheter-related sepsis and central vein thrombosis. We therefore decided to compare the incidence of complications between patients with malignant and non-malignant disease. In addition, as one third of our cancer patients had colo-rectal cancer and these appeared to do better on HPN than patients with other malignancies, we decided to investigate whether this was indeed the case.

Sixty patients who currently are or have been on HPN were categorised on the basis of diagnosis: (1) Non-malignant disease ( $n 40$ cases); (2) Advanced colo-rectal cancer ( $n 7$ cases); (3) Other malignant disease ( $n 13$ cases).

Data regarding the periods spent on HPN, complications and hospital admissions were collected from nutrition support team records and the hospital medical notes.

The BANS 'Form B' was used to categorise patients because of two cases with motility disorders whose underlying malignancy only became apparent well after discharge on HPN. One of these patients had an unusually high incidence of HPN complications related to poor compliance with aseptic technique. Such cases make statistical analysis difficult.

The considered length of time on HPN included from the hospital discharge date until the PN was definitely discontinued, independently of the weekly number of days for which it was prescribed.

The complication leading to an admission was recorded as the only diagnosis for that particular in-patient period, not considering other possible complications developed once the patient was in the hospital, as this could have been influenced by the nosocomial environment. Each in-patient admission was considered as an independent episode.

Analysis of the data showed that patients with a cancer diagnosis had more admissions and spent more than twice as long in hospital per year (on average) after discharge on HPN compared to patients with no malignancy $(P=0.04$ and $P=0.07$, respectively), but there was no difference in the incidence of HPN complications $(P=0.73)$.

Patients with colo-rectal cancer spent on average $60 \%$ less time in hospital than other cancer patients and more time at home following discharge on HPN than patients with other cancers, but this difference was not significant $(P=0.21)$. Colo-rectal cancer patients also had fewer HPN-related complications (both catheter and non-catheter related) than patients in the 'other cancers' group $(P=0.60)$. The statistical power of our analysis, however, could be influenced by the low number of patients and by a few cases which had a disproportionate number of complications.

Survival in the groups with malignancy appears to be longer when there is an ongoing therapeutic option $(P=0.001)$, although whether this reflects the effect of chemotherapy on the disease process or the stage of the disease is uncertain. The majority of patients discharged on HPN for purely palliative reasons survived for longer than 1 month. Deaths occurring at $<1$ month were predicted, but HPN was still judged appropriate for psycho-social reasons.

Further studies are needed to confirm these results.

1. Home Parenteral Nutrition (Adult), Definition No. 12. Specialised Services National Definitions Set, 2nd edition, 2004. Department of Health

2. www.bapen.org.uk e-publication. Annual BANS Report (2006). Artificial Nutrition Support in the UK 2005. 